\title{
Clasificación y Análisis de Métodos para medir Calidad de la Experiencia del Servicio de Televisión sobre Protocolo IP (IPTV)
}

\author{
Juan C. Cuéllar ${ }^{(1)}$, Jesús H. Ortiz(2) y José L. Arciniegas ${ }^{(3)}$ \\ (1) Departamento de Tecnologías de Información y Comunicaciones, Grupo de Investigación en Informática \\ y Telecomunicaciones, Universidad Icesi. Calle 18 \#122-135. Cali-Colombia.(e-mail.jcuellar@icesi.edu.co) \\ (2) Closemobile Research \& Development. Calle Santoña No. 4, piso7, puerta 4, Fuentelabrada-España. \\ (e-mail: jesushamilton.ortiz@gmail.com ) \\ (3) Departamento de Telemática, Grupo de Ingeniería Telemática, Universidad del Cauca. Calle 5 \# 4-70, \\ Popayán, Colombia. (e-mail:jlarci@unicauca.edu.co)
}

Recibido Feb. 13, 2014; Aceptado Abr. 14, 2014; Versión final recibida May. 8, 2014

\begin{abstract}
Resumen
El artículo presenta un análisis documentado de trabajos propuestos por diferentes autores sobre como medir calidad de la experiencia para el servicio de televisión sobre protocolo IP (IPTV). Se analizan principalmente los métodos indirectos, identificando las ventajas y desventajas de cada propuesta. Se presenta un cuadro comparativo entre los diferentes métodos indirectos analizados, permitiendo dar claridad del proceso que se siguió para obtener cada modelo. Algunos modelos indirectos resultan más precisos que otros debido a la cantidad de parámetros de calidad de servicio que utilizan para su generación o porque se basan en pruebas subjetivas obtenidas con anterioridad.
\end{abstract}

Palabras clave: calidad de la experiencia, calidad de servicio, protocolo IP, servicio IPTV

\section{Classification and Analysis of Methods to Measure Quality of Experience of Television on IP Protocol Service (IPTV)}

\begin{abstract}
This article presents a documented analysis of several studies by different authors presented in the literature on how to measure the quality of experience in services of television on IP protocol (IPTV). Indirect methods are especially analyzed identifying the advantages and disadvantages of each proposal. A comparative analysis between the different indirect methods analyzed is presented, giving clarity to the process used to obtain each model. Some indirect models resulted more accurate than others due to the number of parameters of quality service that are used for formulating the model or because they are based on subjective tests previously obtained.
\end{abstract}

Keywords: quality of experience, quality of service, IP protocol, IPTV service 


\section{INTRODUCCIÓN}

El crecimiento actual del servicio de IPTV [Televisión sobre Protocolo IP] hará que, para el 2016, éste corresponda al $88 \%$ del tráfico global de Internet (Cisco, 2012). Esta situación podrá, en determinado momento generar congestión, degradación en los servicios que presten los proveedores de servicio y, por ende, insatisfacción en el usuario final. Es por esto que los proveedores de servicio trabajan ahora en dos frentes, para garantizar que los servicios funcionen de manera óptima: uno de ellos, ofrecer calidad de servicio (Quality of Service [QoS]) en sus redes de núcleo; el otro, estar de manera constante realizando medidas sobre la calidad de experiencia (Quality of Experience [QoE]), entendiéndose esta como la aceptabilidad global de una aplicación o servicio, tal como la percibe subjetivamente el usuario (ITU, 2006), la cual está estrechamente relacionada con la calidad de servicio.

Con base en lo anterior, para los proveedores de servicio es muy importante conocer la calidad de la experiencia de los servicios ofrecidos, con el fin de estar continuamente evaluándolos, no permitir su degradación y, por consiguiente, evitar la insatisfacción en el usuario. Es por esto que la comunidad investigativa ha propuesto una serie de métodos para medir esta calidad de experiencia al servicio de IPTV, y los ha clasificado de la siguiente manera: métodos subjetivos, objetivos e indirectos (Rahrer et al., 2006). Cada uno de ellos tiene su proceso de funcionamiento donde involucra variables de medida y presenta ventajas y desventajas. El objetivo del artículo es presentar los diferentes métodos para evaluar la calidad de experiencia, haciendo especial énfasis en los métodos indirectos, con base en esto el artículo está organizado de la siguiente manera: en la sección métodos para evaluar calidad de la experiencia, se presentan las características de funcionamiento y ejemplos de los métodos subjetivos y objetivos, en la sección métodos indirectos, se presenta un análisis detallado de los diferentes autores que han realizado propuestas bajo este esquema y se presenta una tabla comparativa, al final se presentan las conclusiones, agradecimientos y referencias consultadas.

\section{MÉTODOS PARA EVALUAR CALIDAD DE EXPERIENCIA}

El reporte técnico TR-126 (Rahrer et al., 2006), presenta los métodos para medir o evaluar la calidad de experiencia al servicio de IPTV y clasifica los métodos en: subjetivos, objetivos e indirectos. En esta sección se describirán los métodos subjetivos y objetivos. En la siguiente se analizarán con más detalle los métodos indirectos.

\section{Métodos Subjetivos}

En los métodos subjetivos la medición de la calidad de la experiencia se hace mediante encuestas que se realizan a un grupo de usuarios, generalmente en entornos controlados y siguiendo los lineamientos planteados por la International Telecommunications Union [ITU] (1994; 1997; 2002; 2008); como escala de medición utilizan el MOS (Mean Opinion Score).

La principal ventaja de usar este tipo de método es que se evalúa la calidad de experiencia directamente al usuario y se logran obtener sus atributos sociológicos y psicológicos. La gran desventaja radica en el tiempo y el costo que se debe invertir para realizar las encuestas, y que no se puede hacer para video en línea. Aunque este tipo de método posea estas desventajas es el método utilizado para realizar comprobaciones con los otros métodos con el fin de definir qué tan precisos son.

\section{Métodos Objetivos}

En los métodos objetivos existen dos maneras de clasificar los diferentes algoritmos o formas de medir la calidad de experiencia asociada al video. Una de ellas se basa en la necesidad de utilizar una señal de referencia para realizar la medición y la otra depende del tipo de análisis que se le realice a la traza de video. Con respecto al tipo de medida que requiere la utilización o no de una señal de referencia hay tres enfoques: i) Referencia completa; ii) Referencia reducida; y iii) Sin referencia, que serán explicadas a continuación.

i) Referencia completa: Método en el cual la señal de video original transmitida está disponible en el receptor para comparar la señal original con la recibida y determinar la calidad del video enviado al usuario. Este método entrega buenos resultados en términos de distorsión y correlación, pero no evalúa la calidad absoluta. Es costoso en tiempo porque requiere poder de cómputo para ejecutar algoritmos complejos (Mellouk, Rifai, y Mohammed, 2011; Winkler, 2009); ii) Referencia reducida: método donde está disponible en el receptor información parcial de la señal original transmitida, con el fin de determinar la calidad del video recibido. Este método es más económico en tiempo y recursos que el anterior, pero menos preciso (Mellouk et al., 2011); iii) Sin referencia: método en cual solo se posee la señal de video recibida para 
determinar la calidad del video. La calidad es medida con base en ciertos parámetros definidos que reflejan degradación de la señal, muy útil cuando se desean hacer mediciones en tiempo real (Mellouk et al., 2011).

Considerando los métodos basados en el análisis que se le realice a la traza de video, hay tres tipos de enfoques de medida: medidas basadas en datos, medidas basadas en imagen y medidas basadas en paquetes o flujo de bits (Winkler y Mohandas, 2008).

Con respecto a las medidas basadas en datos se encargan de analizar, pixel a pixel, los cambios de cuadros entre la señal recibida y una señal de referencia. El algoritmo más utilizado que funciona bajo este esquema es el Peak-Signal-to-Noise-Ratio [PSNR] (Winkler y Mohandas, 2008; Wang, 2014; Serral-Graciá et al., 2010).

Por su parte las medidas basadas en imagen, hay dos enfoques, el primero es el modelado de la visión, que también se conoce como enfoque psicológico (You et al, 2010), debido a que se basa en el análisis de varios componentes del sistema visual humano como son: contraste, percepción de color y enmascaramiento temporal y espacial, entre otros. El segundo es el enfoque de ingeniería. Este enfoque de modelado de la visión presenta buen rendimiento ya que los modelos imitan el proceso de formación de la visión humana, pero el proceso de cálculo es muy complejo debido a la complejidad de cada proceso (You et al., 2010). Entre los métodos más utilizados basados en este enfoque se encuentran: Structural Similarity Index [SSIM] (Wang et al., 2004); Multi-scale SSIM [MS-SSIM] (Sheikh et al., 2006); Speed SSIM (Wang y Li, 2007); Video Quality Metric [VQM] (Pinson y Wolf, 2004); Motion-based Video Integrity Evaluation [Movie] (Seshadrinathan y Bovik, 2009); y Visual Signal to Noise Ratio [VSNR] (Chandler y Hemani, 2007).

La desventaja de este tipo de medidas basadas en imagen es que requieren tanto la señal original, como la señal distorsionada, para su análisis, lo que conlleva a que debe existir una sincronización entre el emisor y el receptor, algo bastante complejo de lograr si se desea hacer mediciones en tiempo real; adicionalmente, algunos de los algoritmos mencionados son computacionalmente complejos.

Por otro lado, las medidas basadas en paquetes o flujo de bits analizan la traza de video que es enviada por la red, analizando la información en los encabezados de los paquetes del video codificado. Las grandes ventajas que presenta este esquema son dos: la primera, su bajo requerimiento de ancho de banda, en comparación con las propuestas analizadas hasta ahora; la segunda, que permite analizar varios canales de manera simultánea. Este tipo de medidas tiene cinco modelos de medición: modelos de nivel de red, modelos paramétricos de nivel paquete, modelos paramétricos de planeación, modelos de nivel de flujo de bits y modelos híbridos (Takahashi, Hands, y Barriac, 2008). En la figura 1 se presenta un resumen de los métodos objetivos analizados en esta sección.

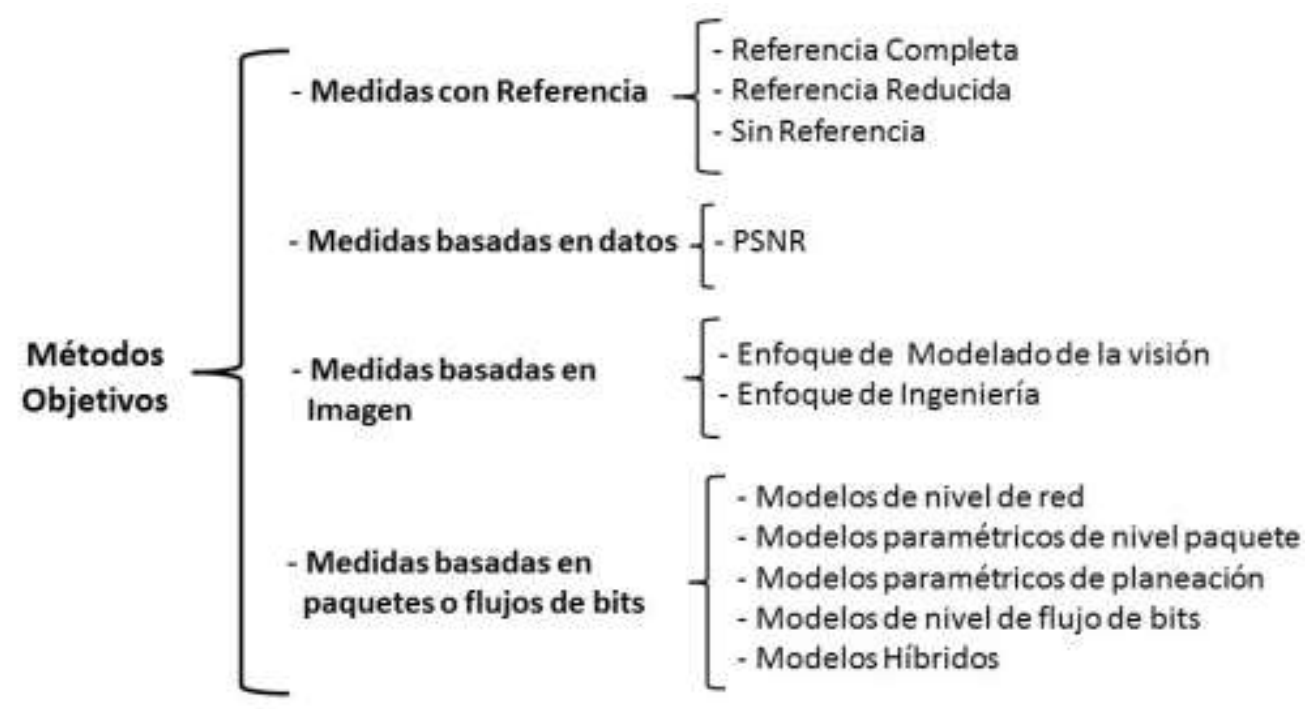

Fig. 1: Cuadro descriptivo de los métodos objetivos para evaluar calidad de experiencia para IPTV.

Por lo tanto ante las desventajas que presentan los métodos subjetivos y objetivos, una solución que permite calcular la calidad de experiencia percibida por un usuario, es mediante la utilización de métodos indirectos. Es por esta razón, que múltiples autores, los presentados en la siguiente sección, han planteado una serie de modelos matemáticos o de correlación para resolver este problema; cada modelo tiene una orientación que depende de la cantidad de parámetros de calidad de servicio que emplea y de los parámetros de la señal de video que incluye para evaluar la calidad de la experiencia. 


\section{MÉTODOS INDIRECTOS}

Estos métodos relacionan parámetros de calidad de servicio (retardo, variación del retardo y pérdida de paquetes, o una combinación de estos) con un método subjetivo u objetivo, para medir calidad de la experiencia. Esta relación del valor de los parámetros de calidad de servicio versus el valor obtenido de calidad de la experiencia es expresada mediante un modelo matemático o un modelo de correlación. En la Figura 1 se puede apreciar el proceso para obtener modelos mediante este enfoque: en el eje $X$ se encuentran los parámetros de calidad de servicio utilizados, y en el eje $\mathrm{Y}$, la medida de calidad de la experiencia obtenida mediante la utilización de un método subjetivo u objetivo. Con ese conjunto de datos, parámetro(s) de calidad de servicio y medida de calidad de la experiencia obtenida, usando el concepto de regresión multi-variable, se obtiene el modelo de correlación.

Con el modelo de correlación definido, ya se puede calcular o predecir la calidad de experiencia del video. Las entradas al modelo de correlación serían los parámetros de calidad de servicio; la salida, será la medición de calidad de experiencia del video analizado, como se aprecia en la Figura 2, al lado derecho.

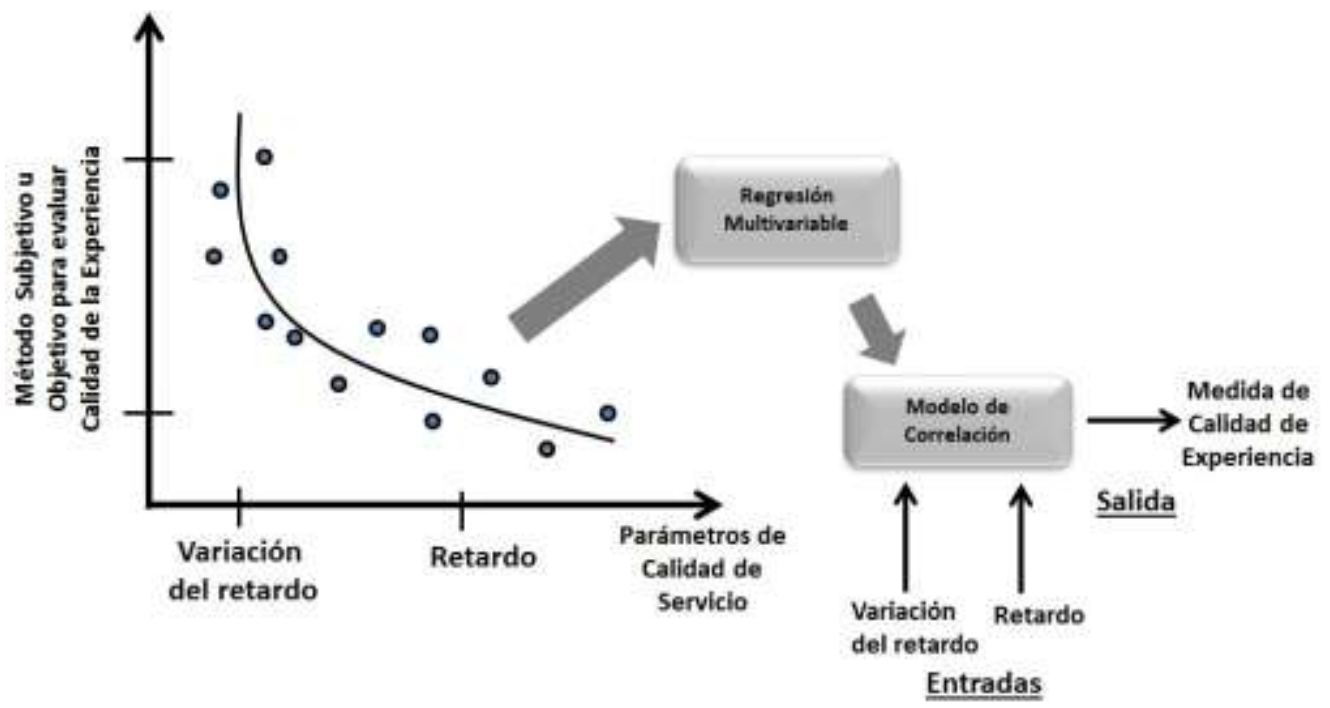

Fig. 2: Proceso para obtener el modelo correlación para medir calidad de experiencia en IPTV.

En el análisis realizado a los autores que han presentados propuestas basándose en métodos indirectos se encontraron tres características procedimentales que permiten establecer una comparación entre cada uno de ellos: proceso o herramienta utilizada, tipo de método o algoritmo utilizado y parámetros de calidad de servicio

Proceso o herramienta utilizada para obtener los datos para generar el modelo: con esta característica se busca definir si el experimento se realizó para obtener datos con personas, utilizando una herramienta de simulación o empleando un montaje de laboratorio; cada manera de obtener los datos presenta ventajas y desventajas.

Tipo de método o algoritmo utilizado para evaluar calidad de la experiencia: esta característica permite indicar el tipo de algoritmo utilizado -subjetivo, objetivo, referencia completa, sin referencia, etc.- para evaluar calidad de la experiencia.

Parámetros de calidad de servicio utilizados: muestra la cantidad de parámetros de calidad de servicio utilizados para generar el modelo.

Tran-Gia et al. (2008) y Fiedler y Hossfeld (2010a) proponen que la relación entre la calidad de servicio y la calidad de la experiencia está dada por la hipótesis IQX (Exponential Interdependency of Quality of Experience and Quality of Service); ellos, mediante pruebas subjetivas analizan cómo se afecta el MOS ante la variación del parámetro pérdida de paquetes. Posteriormente Fiedler y Hossfeld, (2010b) y Zinner et al. (2011) involucran en su modelo tres parámetros de calidad de servicio - medidas de falla, medidas exitosas y medidas de recursos- para analizar con mayor precisión la sensibilidad del usuario, pero se basan en pruebas subjetivas realizadas a un conjunto de usuarios. La limitante de este modelo propuesto con su posterior mejora, radica en que para obtener los datos para generar el modelo, se requiere de pruebas subjetivas realizadas a usuarios. Lo cual costoso en tiempo y en logística debido a que si se desea obtener un conjunto de datos amplio, se requiere realizar un número proporcional de pruebas a los usuarios. 
Lee et al., (2008), Kim y Choi (2010) y Kim et al. (2012) evalúan cinco parámetros de calidad de servicio: perdida de paquetes, nivel de ráfaga, variación del retardo de paquete, retardo de paquete y ancho de banda, asignando a cada parámetro un peso relacionado con su grado de importancia relativa, el cual se obtiene mediante los experimentos presentados por Liu et al. (2006). Mediante una ecuación lineal calculan el valor de calidad de servicio que entregan esos cinco parámetros. Posteriormente, evalúan dicho valor de calidad de servicio en una ecuación exponencial con el fin de obtener el valor de calidad de la experiencia; en esta ecuación involucran tres parámetros adicionales: tamaño de la pantalla, clase de servicio contratado por el usuario y un coeficiente determinado por la estructura de las tramas de acuerdo con el grupo de imágenes Group of Picture [GoP]. Los autores en sus trabajos no especifican como obtienen la ecuación exponencial y adicionalmente utilizan pruebas subjetivas para asignar a cada parámetro su peso relacionado con el grado de importancia del mismo.

Yamagishi et al. (2009) proponen un modelo híbrido sin referencia para estimar la calidad del video, utilizando información de los encabezados de los paquetes y de la señal de video. El modelo estima la degradación de la calidad del video debida a la compresión utilizada en su codificación y la pérdida de paquetes. Los autores obtienen el modelo realizando estudios de correlación de datos obtenidos de la señal de video, analizando información espacial y temporal del mismo. El modelo se basa en pruebas subjetivas, realizadas a un conjunto de videos tomados como ejemplo, y solamente analizan la calidad de la experiencia teniendo como base un solo parámetro de calidad de servicio: la rata de bits. Al igual que modelos presentados con anterioridad requieren de pruebas subjetivas para la obtención de datos y solo emplean como parámetro de calidad de servicio la rata de bits.

Riker et al., (2011) proponen un esquema de predicción hibrido (Hybrid Quality of Experience [HyQoE]) que evalúa el nivel de calidad de un flujo de video automáticamente, en tiempo real, y correlaciona los resultados obtenidos a partir de pruebas subjetivas. El esquema está basado en el aprendizaje estadístico de una red neuronal randómica [RNN] (Random Neural Network) que se entrenó con videos reales generados sobre un escenario simulado inalámbrico. Aunque es una propuesta interesante, requiere del entreno previo de la red neuronal mediante el uso de los datos obtenidos mediante pruebas subjetivas.

Karmounch et al. (2011) presentan un modelo matemático que utiliza una función sigmoid como base para estimar la calidad de la experiencia con respecto a la calidad de servicio percibida por el usuario; la propuesta se basa en el uso de criterio descendente, en el cual la respuesta de la función disminuye, mientras el valor de entrada de la misma aumenta. Para este caso, el valor de entrada de la función es la razón de pérdida de bytes. Así, ante mayor pérdida de bytes, menor valor de calidad de la experiencia percibida por el usuario. Para obtener la relación entre la razón de pérdida de bytes y calidad de la experiencia se utilizó como herramienta de simulación NS-2 y para obtener la calidad de la experiencia percibida por el usuario, índice Mean SSIM [MSSIM] (Sheikh et al., 2004). Esta propuesta no requiere de un alto procesamiento o la utilización de memoria, pero solamente analiza un parámetro de calidad de servicio para obtener la relación de la calidad de la experiencia percibida por parte del usuario.

Mellouk et al. (2012) proponen cuantificar la calidad de la experiencia utilizando varios parámetros de calidad de servicio, basándose en la hipótesis IQX propuesta por Tran-Gia et al. (2008) y Fiedler y Hossfeld (2010). Mellouk et al. (2012) utilizan una regresión lineal entre las diferentes ecuaciones generadas por cada parámetro de calidad de servicio para obtener el modelo. Este modelo utiliza más de un parámetro para evaluar la calidad de la experiencia y contribuye a la solución del problema combinando más de un parámetro de calidad de servicio, pero se basa en la expresión en hipótesis IQX obtenida mediante pruebas subjetivas.

Pokhnel et al. (2013) proponen un modelo para estimar la calidad de experiencia basado en lógica difusa, considerando tres parámetros de calidad de servicio (pérdida de paquetes, pérdida de paquetes en ráfaga y variación del retardo). Para construir el modelo realizan una serie de pruebas subjetivas a un conjunto de 25 usuarios, con el fin de medir la correlación entre los parámetros de calidad de servicio y la calidad de experiencia obtenida al ver los videos utilizados. Como resultado se obtiene un sistema experto difuso que se basa en un método sin referencia para calcular la calidad de experiencia basándose en las condiciones de la red. La gran ventaja que presenta este enfoque es que es simple y requiere poco procesamiento computacional, pero requiere de un gran conjunto de datos obtenidos a partir de pruebas subjetivas...falta adicionar la referencia

Khorsandroo et al. (2012) presentan un enfoque basado en leyes del psicoanálisis para encontrar relaciones cuantitativas a fin de explicar la relación entre la calidad de experiencia y la calidad de servicio. Los autores usan la Ley de Potencia de Steven la cual explica como analizar los cambios de la percepción humana con base en un estímulo físico. En esta relación solo usan un solo parámetro de calidad de servicio para analizar la calidad de experiencia del video. 
En la tabla 1 se presenta un cuadro comparativo entre los autores analizados donde se presenta el proceso o herramienta utilizada para generar el modelo, tipo de método o algoritmo utilizado para medir la calidad de experiencia y parámetros de calidad de servicio usados para generar el modelo, adicionalmente se presentan ventajas y desventajas de cada propuesta. Esta comparación permite apreciar la tendencia en la evolución de los métodos indirectos y también muestra que en la mayoría de los casos métodos subjetivos han sido necesarios para generar los datos que posteriormente se usan para generar y probar los modelos.

Tabla 1: Cuadro comparativo de referentes consultados que utilizan métodos indirectos

\begin{tabular}{|c|c|c|c|c|c|}
\hline Autores & $\begin{array}{l}\text { Herramientas o } \\
\text { proceso utilizado } \\
\text { para obtener los } \\
\text { datos }\end{array}$ & $\begin{array}{l}\text { Tipo de Método y/o } \\
\text { Algoritmo utilizado } \\
\text { para evaluar calidad } \\
\text { de experiencia }\end{array}$ & $\begin{array}{l}\text { Parámetros de } \\
\text { calidad de } \\
\text { servicio utilizados } \\
\text { en el modelo }\end{array}$ & Ventajas & Desventajas \\
\hline $\begin{array}{l}\text { Tran-Gia et al., } \\
\text { (2008), Fiedler y } \\
\text { Hossfeld (2010a; } \\
\text { 2010b), Zinner et } \\
\text { al., 2011). }\end{array}$ & $\begin{array}{l}\text { Montaje en } \\
\text { laboratorio } \\
\text { MOS }\end{array}$ & $\begin{array}{l}\text { Pruebas subjetivas } \\
\text { utilizando hipótesis } \\
\text { IQX (Exponential } \\
\text { Interdependency of } \\
\text { QoE and QoS) }\end{array}$ & $\begin{array}{l}\text { Pérdida de } \\
\text { paquetes } \\
\text { Variación del } \\
\text { retardo. }\end{array}$ & $\begin{array}{l}\text { Es uno de los autores } \\
\text { referentes en este tema y } \\
\text { es tomado como base } \\
\text { para muchas otras } \\
\text { publicaciones }\end{array}$ & $\begin{array}{l}\text { Utiliza muy pocas } \\
\text { variables y se basa } \\
\text { en datos subjetivos } \\
\text { para obtener el } \\
\text { modelo }\end{array}$ \\
\hline $\begin{array}{l}\text { Lee et al., (2008), } \\
\text { Kim y Choi } \\
(2010) \text {, Kim et al., } \\
2012) .\end{array}$ & No está definido & $\begin{array}{l}\text { Ambiente de } \\
\text { computación invasiva } \\
\text { (Liu et al., 2006) }\end{array}$ & $\begin{array}{l}\text { Perdida de } \\
\text { paquetes, nivel } \\
\text { de ráfaga, varia- } \\
\text { ción del retardo } \\
\text { de paquete, } \\
\text { retardo de } \\
\text { paquete y ancho } \\
\text { de banda. }\end{array}$ & $\begin{array}{l}\text { Utiliza un número superior } \\
\text { de parámetros a los } \\
\text { usados por otros autores } \\
\text { e incluye aspectos de } \\
\text { configuración del servicio } \\
\text { que recibe el usuario final. }\end{array}$ & $\begin{array}{l}\text { El peso asignado a } \\
\text { cada parámetro de } \\
\text { calidad de servicio no } \\
\text { es claro, ni tampoco } \\
\text { la manera de obtener } \\
\text { el modelo propuesto. }\end{array}$ \\
\hline $\begin{array}{l}\text { Yamagish et al. } \\
\text { (2009). }\end{array}$ & $\begin{array}{l}\text { Montaje en } \\
\text { laboratorio } \\
\text { Pruebas subjetivas }\end{array}$ & $\begin{array}{l}\text { Correlación de } \\
\text { parámetros }\end{array}$ & $\begin{array}{l}\text { Perdida de } \\
\text { paquetes }\end{array}$ & $\begin{array}{l}\text { Analiza la información de } \\
\text { los encabezados de los } \\
\text { paquetes, sin tomar en } \\
\text { cuenta la información } \\
\text { debida a la codificación y } \\
\text { compresión usada para } \\
\text { transmitir el video }\end{array}$ & $\begin{array}{l}\text { Utiliza solo un } \\
\text { parámetro para } \\
\text { construir el modelo } \\
\text { basándose en } \\
\text { pruebas subjetivas } \\
\text { realizadas a un grupo } \\
\text { de ususarios }\end{array}$ \\
\hline $\begin{array}{l}\text { Riker et al., } \\
\text { (2011). }\end{array}$ & $\begin{array}{l}\text { Utilizan una red } \\
\text { Neuronal } \\
\text { Randómica }\end{array}$ & $\begin{array}{l}\text { Entrenan a la red con } \\
\text { videos evaluados } \\
\text { subjetivamente }\end{array}$ & $\begin{array}{l}\text { No utiliza. La Red } \\
\text { Neuronal se } \\
\text { entrena con } \\
\text { videos previa- } \\
\text { mente evaluados }\end{array}$ & $\begin{array}{l}\text { La gran ventaja de esta } \\
\text { propuesta es que funciona } \\
\text { en tiempo real. }\end{array}$ & $\begin{array}{l}\text { Es necesario entre- } \\
\text { nar la red neuronal } \\
\text { con videos que han } \\
\text { sido previamente } \\
\text { evaluados de manera } \\
\text { subjetiva. }\end{array}$ \\
\hline $\begin{array}{l}\text { Karmounch et al., } \\
\text { (2011). }\end{array}$ & Simulador NS-2 & Función Sigmoid & $\begin{array}{l}\text { Proporción de } \\
\text { pérdida de bytes. }\end{array}$ & $\begin{array}{l}\text { Este método no requiere } \\
\text { gran procesamiento o alto } \\
\text { consumo de memoria. }\end{array}$ & $\begin{array}{l}\text { Solo utiliza un solo } \\
\text { parámetro de calidad } \\
\text { de servicio. }\end{array}$ \\
\hline $\begin{array}{l}\text { Mellouk et al., } \\
\text { (2012). }\end{array}$ & $\begin{array}{l}\text { Montaje en } \\
\text { laboratorio MOS }\end{array}$ & $\begin{array}{l}\text { IQX y Regresión } \\
\text { Lineal Multivariable }\end{array}$ & $\begin{array}{l}\text { Retardo y pérdida } \\
\text { de paquetes }\end{array}$ & $\begin{array}{l}\text { Permite la incorporación } \\
\text { de varios parámetros de } \\
\text { calidad de servicio al } \\
\text { modelo. }\end{array}$ & $\begin{array}{l}\text { Los autores basan su } \\
\text { trabajo en Fiedler y } \\
\text { Hossfeld (2010a), el } \\
\text { cual es basado en } \\
\text { pruebas subjetivas. }\end{array}$ \\
\hline $\begin{array}{l}\text { Pokhnel et al. } \\
\text { (2013) }\end{array}$ & $\begin{array}{l}\text { Pruebas subjetivas } \\
\text { a un grupo de } \\
\text { usuarios }\end{array}$ & $\begin{array}{l}\text { Sistema experto } \\
\text { basado en lógica } \\
\text { difusa }\end{array}$ & $\begin{array}{l}\text { Pérdida de } \\
\text { paquetes, pérdida } \\
\text { de paquetes en } \\
\text { ráfaga y variación } \\
\text { del retardo }\end{array}$ & $\begin{array}{l}\text { Analiza diferentes tipos } \\
\text { videos ( basados en } \\
\text { contenido) para construir } \\
\text { el modelo y utiliza tres } \\
\text { parámetros de calidad de } \\
\text { servicio. }\end{array}$ & $\begin{array}{l}\text { Se requiere de un } \\
\text { grupo de usuarios } \\
\text { para realizar las } \\
\text { pruebas subjetivas } \\
\text { para obtener los } \\
\text { datos para construir } \\
\text { el modelo. }\end{array}$ \\
\hline $\begin{array}{l}\text { Khorsandroo et } \\
\text { al. (2012) }\end{array}$ & $\begin{array}{l}\text { Modelo adaptado } \\
\text { de leyes del } \\
\text { psicoanálisis }\end{array}$ & Relación de potencia & $\begin{array}{l}\text { Pérdida de } \\
\text { paquetes }\end{array}$ & $\begin{array}{l}\text { El modelo se basa en una } \\
\text { ley de psicoanálisis, la } \\
\text { cual tiene una } \\
\text { fundamentación teórica e } \\
\text { investigativa claramente } \\
\text { documentada }\end{array}$ & $\begin{array}{l}\text { El modelo utiliza un } \\
\text { parámetro de calidad } \\
\text { de servicio, para } \\
\text { verificar la respuesta } \\
\text { de calidad de } \\
\text { experiencia obtenida. }\end{array}$ \\
\hline
\end{tabular}

\section{CONCLUSIONES}

La comunidad científica ha trabajado con base en tres clases de métodos para realizar la medición de la calidad de experiencia para IPTV, métodos subjetivos, objetivos e indirectos. Los métodos subjetivos se han convertido en un estándar de facto para realizar las mediciones, pero son costosos en recursos, tiempo y dinero, debido a la logística que demanda su realización, pues se debe tener un ambiente adecuado y una población de características especiales, con el fin de lograr mediciones lo más reales posible. Por su parte, los métodos objetivos tratan de superar las limitaciones presentadas en los métodos subjetivos, pero con un costo computacional alto; algunos, además, requieren de la señal origen para establecer comparaciones y así obtener la medida de calidad de experiencia.

Ante esto, la comunidad científica ha propuesto, en un lapso de tiempo relativamente corto, modelos para la medición de la calidad de experiencia basándose en métodos indirectos. Estos métodos indirectos generalmente conducen a un modelo de correlación intentan contribuir a la solución del problema utilizando como base datos obtenidos en pruebas subjetivas o datos obtenidos mediante pruebas de laboratorio 0 
herramientas de simulación. Los modelos propuestos buscan que las mediciones de calidad de experiencia se puedan realizar en línea, con el fin de obtener, de manera automática, la medición de calidad de experiencia, y así realizar los correctivos necesarios sobre la infraestructura de red o sobre la generación de la señal de video.

Algunos modelos indirectos son más precisos que otros debido a la cantidad de parámetros de calidad de servicio que utilizan para su generación; otros, se basan en pruebas subjetivas obtenidas con anterioridad. Este tipo de situaciones hace que algunos modelos propuestos tengan desventajas frente a otros que obtienen sus datos mediante pruebas de laboratorio o utilizando alguna herramienta de simulación.

\section{AGRADECIMIENTOS}

Los autores agradecen al Programa Interamericano de Ciencia y Tecnología para el Desarrollo [CYTED] por el apoyo a través del proyecto Red temática REDAUTI en aplicaciones y usabilidad de la televisión digital interactiva [código 512RT0461]. Igualmente agradecen a la Facultad de Ingeniería de la Universidad de Icesi por su apoyo en el marco del proyecto Modelo para la Medición de QoE para el servicio de IPTV.

\section{REFERENCIAS}

Chandler, D. y Hemani, S. VSNR: A wavelet-based visual signal-to-noise ratio for natural images. IEEE Transactions on Image Processing, 16(9), 2284-2298 (2007).

Cisco. (2012, mayo 30). Cisco visual networking index: Forecast and methodology, 2011-2016. San Jose, CA: Cisco. Recuperado en enero 4, 2014, de Cisco:

http://www.cisco.com/en/US/solutions/collateral/ns341/ns525/ns537/ns705/ns827/white_paper_c11481360.pdf

Fiedler, T. y Hossfeld, M. A generic quantitative relationship between quality of experience and quality of service. IEEE Network, 24(2), 36-41 (2010a).

Fiedler, T. y Hossfeld, M. Quality of experience-related differencial and provisioning-delivery hysteresis. ITCSS21. Tokyo, Japón: IEICE. (2010b).

ITU, International Telecommunications Union, Recommendation ITU-R BS.775-1, Multichannel sterophonic sound system with and without accompanying picture. Ginebra, Suiza (1994).

ITU, International Telecommunications Union, Recommendation BS.1286. Methods for the subjective assessment of audio systems with accompanying picture. Ginebra, Suiza: ITU (1997).

ITU, International Telecommunications Union [ITU]. ITU-R Recommendation BT.500-11. Methodology for subjective assessment of the quality of television pictures. Ginebra, Suiza: ITU (2002).

ITU, International Telecommunications Union [ITU]. ITU-T Recommendation G.1080. Quality of experience requerimentes for IPTV services. Ginebra, Suiza: ITU. (2006).

ITU, International Telecommunications Union [ITU]. ITU-T Recommendation P.910. Subjective video quality assesment methods for multimedia applications. Ginebra, Suiza: ITU (2008).

Karmounch, A., Abdeljaouad, I., y Kandavanam, G. A Loss-based Utility Function for Predicting IPTV Quality of Experience over an Overlay Netowork. Global Telecommunications Conference (GLOBECOM 2011), 2011 IEEE (pp. 1-6). Piscataway, NJ: IEEE (2011).

Kim, H.-J., Yun, D.-G., Kim, H.-S., Cho, K.-S., y Choi, S.-G. QoE assessment model for video streaming service using QoS parameters in wired-wireless network. Advanced Communication Technology (ICACT), 2012 14th International Conference on (pp. 459-464). Seul, Corea: Global IT Research Institute. (2012).

Kim, S. y Choi, H. A Study on a QoS/QoE correlation model for QoE evaluation on IPTV service. Advanced Communication Technology (ICACT), 2010 The 12th International Conference on (v2, pp. 1377-1382). Seul, Corea: Electronics and Telecomm. Research Institute / National Information Society Agency (2010).

Khorsandroo S., Noor R. M., and Khorsandroo S., "The Role of Psychophysics Laws in Quality of Experience Assessment: A Video Streaming Case Study," in Proceedings of the International Conference on Advances in Computing, Communications and Informatics, New York, NY, USA, pp. 446-452. (2012).

Lee, J., Lee, K., Lyu, W., Choi, S., Kim, H., y Lee, D. The QoE evaluation method through the QoS-QoE correlation model. Networked Computing and Advanced Information Management, 2008. NCM '08. Fourth International Conference on (v2, pp. 719-725). Piscataway, NJ: IEEE. (2008). 
Liu, L.-y., Zhou, W.-a., y Song, J.-d. The research of quality of experience evaluation method in pervasive computing environment. Pervasive Computing and Applications, 2006 1st International Symposium on (pp. 172-182). Piscataway, NJ: IEEE (2006).

Mellouk, A., Aroussi, S., y Bouabana, T. Empirical QoE/QoS correlation model based on multiple parameters for VoD flows. Global Communications Conference (GLOBECOM), 2012 (1963-1968). Piscataway, NJ: IEEE (2012).

Mellouk, A., Rifai, H., y Mohammed, S. A Brief Syntehsis of QoS-QoE Methodologies. Programming and Systems (ISPS), 2011 10th International Symposium on (pp. 32-38). Piscataway, NJ: IEEE (2011).

Pinson, M. y Wolf, S. A new standardized method for objectively measuring video quality. Broadcasting, IEEE Transactions on, 50(3), 312-322 (2004).

Pokhrel J., Wehbi B., Morais A., Cavalli A., and Allilaire E., "Estimation of QoE of video traffic using a fuzzy expert system," in 2013 IEEE Consumer Comm. and Networking Conference (CCNC),pp. 224-229 (2013).

Rahrer T.,Fiandra R.,Wright S. [Eds.]. Technical Report TR-126: Triple Play Services Quality of Experience (QoE) Requirements. Recuperado en enero 3, 2014, de http://www.broadbandforum.org/technical/download/TR-126.pdf (2006).

Riker, A., Aguiar, S., Cardoso, D., Oliveira, P., Cerqueira, E., y Abelém, A. A hybrid prediction and assessment quality of experience approach for videostreaming applications over wireless netowrks. XXIX Simpósio Brasileiro de Redes de Computadores e Sistemas Distribuídos (pp. 367-380). Campo Grande, Brasil: Universidade Federal de Mato Grosso do Sul (2011).

Serral-Graciá, R. y otros 5 autores. An overview of quality of experience measurement challenges for video applications in IP networks. WWIC'10 Proceedings of the 8th international conference on Wired/Wireless Internet Communications. Springer-Verlag, pp. 252-263. Heidelberg, Alemania: Springer-Verlag (2010).

Seshadrinathan, K. y Bovik, A. Motion-based perceptual quality assessment of video [en línea]. Recuperado en enero 4, 2014, de University of Texas. Digital Repositorie: http://repositories.lib.utexas.edu/handle/2152/ 17765 (2009).

Sheikh, H., Sabir, M., y Bovik, A. A Statistical evaluation of recent full reference image quality assessment algoritms. Image Processing, IEEE Transactions on, 15(11), 3340-3451 (2006).

Sheikh, R., Wang, Z., Bovik, A., y Simoncelli, P. Image quality assessment: From error visibility to structural similarity. Image Processing, IEEE Transactions on, 13(4), 600-612 (2004).

Takahashi, A., Hands, D., y Barriac, V. Standardization activities in the ITU for a QoE assessment of IPTV. IEEE Communications Magazine, 46(2), 78-84 (2008).

Tran-Gia, P., Tutschku, K., Fiedler, M., Hossfeld, T., y Hock, D. Testing the IQX hypothesis for exponential iinterdependency between QoS and QoE of voice codecs Ilbc adn G711 [Report No.442]. Wurzburg, Alemania: University of Wurzburg (2008).

Wang, Y., Survey of objetive video quality measurements. Recuperado en enero 4, 2014, de ftp://ftp.cs.wpi.edu/pub/techreports/pdf/06-02.pdf

Wang, Z. y Li, Q. Video Quality Assessment Using a Statistical Model of Human visual Speed Peception. $J$ Opt Soc Am A Opt Image Sci Vis, 24(12), B61-B69. (2007).

Wang, Z., Lu, L., y Bovik, A. Video quality assessment based in structural distortion measurement. Signal Processing: Image Communication, 19(2), 121-132. (2004).

Winkler, S. Video quality measurement standards - Current status and trends. Information, Communications and Signal Processing, 2009. ICICS 2009. 7th International Conference on (pp. 1-5). 7th International Conference on Information, Communications and Signal Processing.: Piscataway, NJ (2009).

Winkler, S. y Mohandas, P. The evolution of video quality measurement: From PSNR to hybrid metrics. IEEE Transactions on Broadcasting, 54(3), 660-668 (2008).

Yamagishi, K., Kawano, T., y Hayashi, T. Hybrid video-quality-estimation model for IPTV services. Global Telecommunications Conference, 2009. GLOBECOM 2009. IEEE (pp. 1-5). Piscataway, NJ: IEEE (2009).

You, J., Reiter, U., Hannuksela, M., Gabbouj, M., y Perkis, A. Perceptual-based quality assessment for audio-visual services: A survey. Signal Processing: Image Communication, 25(7), 482-501 (2010).

Zinner, T., Hossfeld, T., y Fiedler, M. The QoE provisioning-delivery hysteris and its importance for service provisioning in the future. Next Generation Internet (NGI), 2011 7th EURO-NGI Conference on (pp. 1-8). Piscataway, NJ: IEEE (2011). 